\title{
TCM Treatment of Polycystic Ovaries and PCOS Using Integrated Medical Diagnosis---- 58 Cases Study
}

\author{
Dan Jiang* \\ Principal and TCM Consultant in Hallam Institute of TCM, UK
}

Submission: April 20, 2021; Published: May 25, 2021

*Corresponding author: Dan Jiang, Principal and TCM Consultant in Hallam Institute of TCM, UK

\begin{abstract}
Introduction: Polycystic ovary syndrome is a disease from which recent reports show one in five women of reproductive age suffer. The author distinguishes between polycystic ovaries (PCO) as a preclinical condition, or a gentle stage which manifests as prolonged menstrual cycles, even amenorrhea at times, and polycystic ovary syndrome (PCOS), a typical disease which manifests as a sexual hormonal disorder. Both PCO and PCOS are becoming more widespread and have become common causes of infertility. Most cases manifest as PCO in varied degrees, severe cases of PCOS being less common. Using the TCM diagnostic approach, the author identifies three patterns in PCO and PCOS: Qi deficiency of spleen and kidney, with accumulation of fluid and stasis of blood; Kidney Yang deficiency and accumulation of fluid and phlegm; Liver Qi stagnation and blood stasis. She proposes an effective treatment for each pattern, according to the TCM clinical approach, combining acupuncture with Chinese herbal medicine, which leads to the successful regulation of the menstrual cycle and assisted pregnancies. Treatment methods and typical cases treated in UK have been summarized and reported.
\end{abstract}

Conclusion: Using diagnostic references from Conventional Western Medicine (CWM) can raise the efficacy of TCM methods. The advantage of TCM lies in the treatment of the preclinical state or gentle stage of a serious disease, thus preventing it from progressing to severe stage of the disease. This combined approach has resulted in many patients regaining regular menstrual cycles and subsequently successfully conceiving and giving birth.

Keywords: Polycystic ovaries; Polycystic ovary syndrome; Infertility; TCM

\section{Polycystic Ovaries and PCOS}

Polycystic ovaries are characterized by the appearance of numerous small cysts on the ovaries which are diagnosed by an ultrasound scan. Polycystic ovaries can cause infrequent and prolonged menstruation, occasionally amenorrhea, although sometimes there are no symptoms at all. It is usually diagnosed at a regular infertility examination. The latest reports show that around one in five women suffer from polycystic ovaries $[1,2]$.

Polycystic ovary syndrome (PCOS) is a disease which is caused by the disorder of sexual hormones. Its main symptoms are amenorrhea, infertility, habitual miscarriage, obesity, hirsutism (excess of facial and body hair) and virilism (secondary male sexual characteristics). It is caused by oligo ovulation which means the ovaries can't produce a sufficient number of mature eggs and at times fail to ovulate. I believe that polycystic ovaries is the early, or milder stage of PCOS; it belongs to a preclinical condition. TCM can treat and successfully cure PCO, using acupuncture and herbal medicines. PCOS, however, is an organic disease, for which it is important to carefully identify the correct pattern, so that a longer and more comprehensive treatment can be determined which will lead to a successful outcome.

\section{The cause of polycystic ovaries and PCOS}

\section{Normal ovulation}

There is a sexual hormonal axis in the female reproductive system which involves the hypothalamus, pituitary and ovaries. In a normal cycle, the hypothalamus gland at the base of the brain produces a hormone called gonadotropin release hormone (GnRH) which stimulates the pituitary to release two hormones: follicle stimulating hormone (FSH) and luteinizing hormone (LH). Both these hormones have a direct effect on the ovaries during the 
menstrual cycle. FSH stimulates the growth of small sacs in the ovaries, (one of which will mature to a follicle containing an egg), as well as producing additional hormones. LH supports FSH to stimulate the follicle, enabling the egg to become mature and then released, ready for fertilization by sperm, both before and after ovulation. In the ovary a small egg cell is gradually developing into a mature follicle, which comes into ovulation around the 14th day of the menstrual cycle. This mature egg swims to the uterus by passing through the fallopian tubes. If it is fertilized by sperm, it will develop into a pregnancy; if the egg is not fertilized, the endometrium will shed as a menstrual period 14 days after ovulation. Thus, a perfect menstrual cycle lasts 28 days [3].

\section{Polycystic Ovaries and PCOS}

An ultrasound scan will show that a patient with polycystic ovaries (PCO) has numerous small cysts, usually no bigger than $8 \mathrm{mms}$, on the ovaries. A normal follicle should be $20 \mathrm{~mm}$ in diameter. The blood test of a patient with PCO or PCOS will show elevated levels of LH and an imbalanced LH / FHS ratio. The follicle's slower growth and maturity may be due to elevated LH or low LH vitality. In a normal menstrual cycle, it takes 14 days for ovulation to take place; in patients with PCO it can take 18-20 days or more. If LH levels are very elevated, or LH vitality very low, the patient will manifest oligo ovulation or even amenorrhea. (Some women may have their period, but no egg in it). Polycystic ovaries can occur in women in all fertility ages and may develop to PCOS at any point $[4,5]$.

\section{The Causes of Slow Ovulation and Oligomenorrhoea}

a) Heredity: most women who suffer from PCO and PCOS come from families whose female members suffer from with the same disorders. This may be caused by the impairment of a gene.

b) Discontinued use of oral contraceptive pills (estrogen type) may cause hormonal disorders or decrease the vitality of LH.

c) A prolonged period of stress or depression can cause disorder of the sexual axis.

d) Environmental or sudden climate changes can influence the hormonal system.

e) Some endocrinal diseases, such as hyperthyroidism or hypothyroidism, can trigger sexual hormonal disorders.

\section{Clinical Symptoms of Polycystic Ovaries and PCOS}

Conventional Western Medicine (CWM) defines polycystic ovaries as the occurrence of numerous cysts on the ovaries, with no more clinical symptoms. Clinical symptoms, such as amenorrhea, obesity, appearance of secondary male sexual characteristics, etc. lead to a diagnosis of PCOS. In practice, however, some patients are diagnosed with PCOS with very mild symptoms. The author believes it is of key importance to make timely diagnoses of both disorders, whilst clinically distinguishing between them, so that TCM treatment can be started as early as possible.

\section{Clinical Features of Polycystic Ovaries}

a) Irregular or regular prolonged menstrual cycle, or occasional amenorrhea which is not over 2-3 months in general. Normal or light periods, lasting 4-6 days without dysmenorrhea (painful periods).

b) Possible infertility.

c) Many small cysts (immature follicles) are found in the ovaries by ultrasound scan.

d) Blood test results normal or slightly increased levels of LH.

\section{Clinical Features of Polycystic Ovary Syndrome}

a) Irregular menstruation: a prolonged menstrual circle, continuous amenorrhea (more than 3 months), heavy periods.

b) Infertility.

c) Tendency towards miscarriages during pregnancy.

d) Hirsutism (excess of facial and body hair), acne on the face, chest, arms and legs.

e) Obesity, the BMI (Body Bass Index) over $25 \mathrm{~kg} / \mathrm{m}$

f) Ultrasound scan: large ovaries with more than 10 cysts (immature follicles) are

g) found.

h) Blood tests show elevated $\mathrm{LH}$, imbalance of $\mathrm{LH} / \mathrm{FH}$ ratio, elevated testosterone $[2,6]$.

\section{CWM Routine Treatment}

CWM prescribes the concentrative pills to women who do not wish to become pregnant and Clomifene Citrate 50-100mg or some other ovulation stimulant to women who wish to become pregnant. Doctors are disinclined to suggest IVF treatment to patients with PCOS as they would be at risk of ovarian hyperstimulation syndrome.

\section{TCM's Perspective on Polycystic Ovaries and PCOS}

Prolonged periods and amenorrhea being the main complaints, TCM recognizes both polycystic ovaries and PCOS as the patterns of Qi and Yang deficiencies, accumulated phlegm-fluid and blood stasis. The common patterns are indicated as below:

\section{Qi Deficiency of Spleen and Kidney, Accumulation of Fluid and Stasis of Blood}

This pattern is often seen in cases with the early, milder stage of polycystic ovaries which may manifest as a longer menstrual cycle, fatigue, pale tongue with teeth marks and white-slippery coating, and a rolling-fine pulse.

Analysis: The following may be causes of the disorder: excessive physical work, major changes in life, a weak constitution 
after a long term of illness, or a miscarriage. The patient appears in an overall weak condition. The spleen and kidney Qi deficiencies result in a failure to transport fluids. The accumulated fluid obstructs the Qi and blood flowing in the uterus, causing disharmony of the menstrual cycle which then lasts longer. Severe accumulation of the Qi-fluid and blood stasis in the uterus can lead to amenorrhea.

\section{Kidney Yang Deficiency and Accumulation of Fluid and Phlegm}

This pattern is the commonest one, with a prolonged menstrual cycle, amenorrhea, cold limbs, aversion to cold, darkened complexion, acne on the face, chest and back, sometimes obesity, swelling at lower legs, excess of hair, heavy feeling in general. Pale tongue with white-slippery coating and a deep-rolling pulse should be manifested.

Analysis: This pattern is often seen in cases with a congenital weakness, or after long term use of oral contraceptive pills which restrain the Kidney Qi. The kidney Qi fails to keep fluids flowing which then remain in the kidneys for longer, thus damaging the kidney Yang. The Kidney Yang fails to sufficiently warm the uterus to produce blood stasis in it. Cold and stasis in the uterus bring about a longer menstrual circle, in some cases amenorrhea and infertility. Fluids are accumulated for longer and become phlegm; fluid-phlegm remains under the tissue causing obesity and obstructing defensive Qi to cause aversion to cold. Patient feels a general heaviness and has cold limbs.

\section{Liver Qi Stagnation and Blood Stasis}

The main symptoms are: stress, agitation, depression, restlessness, weepiness, swollen breasts and dysmenorrhea; acne on cheeks, headaches, prolonged menstrual cycles, in some cases amenorrhea, infertility; light red tongue with thin white coating, wiry, or wiry-fine pulse.

Analysis: This pattern is often seen in women suffering from work related stress and dissatisfaction with their lives, making the Liver Qi move slowly with a tendency to stagnate. Other causes can be: miscarriage, an artificial termination of a normal pregnancy which often leaves the patient struggling to recover her Liver and Kidney Qi; hormonal treatment before an IVF, which has an adverse effect on the patient's hormonal system with the added frustration following a failed IVF treatment. All of the above reasons can cause liver Qi stagnation and blood stasis in the uterus which lead to a sluggish menstrual ccle, with the possibility of amenorrhea. As the liver then fails to keep the Qi moving, patients are often ver -emotional and have mental health symptoms.

\section{TCM Treatment}

With a clear CWM diagnosis, the author believes it is possible to offer a more specialized, and therefore, more accurate TCM treatment plan focused on PCO or PCOS. Using CWM references, also allows us to predict with greater confidence the outcome of
CTM treatment.

\section{Acupuncture:}

a) Main points: Baihui (Du20), Moxi at Shenque (Ren8)

Zhongji (Ren3), or Guanyuan (Ren4), Qihai (Ren6)

Guilai (St28) or Zigong (Ext)

\section{b) Associated points:}

a) Strengthen the spleen and remove the excessive fluid:

Taibai (Sp3)

$$
\text { Xuehai (Sp10), Yinlingquan (Sp9), Sanyinjiao (Sp6), }
$$

b) Replenish the kidneys and warm Yang:

(Ki6), Taixi (Ki3)

Yingu (Ki10), Zusanli(St36), Fuliu (Ki7), Zhaohai

c) Remove the Liver Qi and dissolve the blood stasis:

Waiguan (Sj5), Zulinqi (Gb41)

Hegu (Li4), Taichong (Liv3)

The acupuncture points' formula for each person will be determined according to the specific information on the individual case.

Herbal Medicine: (Table 1) In cases with many symptoms or ones with a complex pattern, a prescribed menstrual regulatory treatment with herbal medicine will be determined [5].

\section{Clinical Summary}

General: I have summarized 58 cases of women who have been diagnosed with or suspected to have* PCO or PCOS, with irregular menstrual cycles, amenorrhea and infertility as the main complaints. All these cases were treated at my clinic from January 2015 to January 2021. *Patients manifest clinical symptoms, but do not have a formal diagnosis by Gynecologists, and nor ultrasound or blood test results to confirm it. Patients 'characteristics:

Age

The youngest 15 , the oldest 50 ;

<20: 4 cases; 21-30: 11cases; 31-40: 35 cases; >41: 8 cases

Fertility age: 54 cases

Main complaints: (Table 2)

\section{Diagnosis}

Polycystic ovaries: 46 (79\%)

Polycystic ovary syndrome: 12 (21\%) 
Table 1: TCM's patterns and relevant herbal formulas (4).

\begin{tabular}{|c|c|c|c|}
\hline Patterns & \multicolumn{2}{|c|}{ Herbal Formula with Main Ingredients } & Herbal Pills \\
\hline \multirow[b]{2}{*}{$\begin{array}{l}\text { Qi deficiency of spleen and kidney, } \\
\text { and accumulation of fluid and stasis } \\
\text { of blood }\end{array}$} & \multicolumn{2}{|c|}{ Sheqi wan plus, Cangfudaotan Tang } & \multirow[b]{2}{*}{$\begin{array}{c}\text { Jinguishenqi Wan, } \\
\text { Renshenguipi Wan, } \\
\text { Banxiatianmabaizhu Wan, } \\
\text { Erchen Wan }\end{array}$} \\
\hline & $\begin{array}{c}\text { Chinese Spelling } \\
\text { Shedihuang, } \\
\text { Shanzhuyu, Shanyao, } \\
\text { Duzhgong, } \\
\text { Tusizi, } \\
\text { Mudanpi, } \\
\text { Zexie, } \\
\text { Fuling, } \\
\text { Xiangfu, } \\
\text { Canzhu, } \\
\text { Banxia. }\end{array}$ & $\begin{array}{c}\text { Ladin Name } \\
\text { Radix Rehmanniae } \\
\text { Fructus Corni } \\
\text { Rhizoma Dioscoreae } \\
\text { Cortex Eucommiae } \\
\text { Semen Cuseutae; } \\
\text { Cortex Moutan } \\
\text { Rhizoma Alismatis } \\
\text { Poria } \\
\text { Rhizoma Cyperi } \\
\text { Rhizoma Atractylodis } \\
\text { Rhizoma Pinelliae }\end{array}$ & \\
\hline \multirow[b]{2}{*}{$\begin{array}{l}\text { Kidney Yang deficiency and accumu- } \\
\text { lation of fluid and phlegm }\end{array}$} & \multicolumn{2}{|c|}{ ErxianTang plus guizhifuling Wan: } & \multirow[b]{2}{*}{$\begin{array}{c}\text { Yougui Yin, } \\
\text { Baifeng Wan, } \\
\text { Nuangongyunzi Wan, } \\
\text { Guizhifuling Wan }\end{array}$} \\
\hline & $\begin{array}{l}\text { Chines spelling } \\
\text { Xianmao, } \\
\text { Yinyanghuo, } \\
\text { Gouqizi, } \\
\text { Bajitian, } \\
\text { Aiye, } \\
\text { Banxia, } \\
\text { Taoren, } \\
\text { Honghua, } \\
\text { Guizhi, } \\
\text { Fuling, } \\
\text { Chuanxiong }\end{array}$ & $\begin{array}{c}\text { Latin Name } \\
\text { Rhizoma Curculiginis } \\
\text { Herba Epimedii } \\
\text { Fructus Lycii } \\
\text { Radix Morindae Officinalis } \\
\text { Folium Artemisiae Argyi } \\
\text { Rhizoma Pinelliae } \\
\text { Semen Persicae } \\
\text { Flos Carthami } \\
\text { Ramulus Cinnamomi } \\
\text { Poria } \\
\text { Rhizoma Chuanxiong }\end{array}$ & \\
\hline & \multicolumn{2}{|c|}{ Chaihu Ji plus Taohongsiwu Wan: } & \multirow[b]{2}{*}{$\begin{array}{l}\text { Chaihushugan Wan, } \\
\text { Xiaoyao /Jiaweixiaoyao wan, } \\
\text { Xuefuzhuyu Wan, } \\
\text { Taohongsiwu Wan }\end{array}$} \\
\hline Liver Qi Stagnation and blood stasis & $\begin{array}{l}\text { Chinese Spelling } \\
\text { Chaihu, } \\
\text { Baishao, } \\
\text { Danggui, } \\
\text { Zhiqiao, } \\
\text { Chishao, } \\
\text { Shengdi, } \\
\text { Chuanxiong, } \\
\text { Yimucao, } \\
\text { Mudanpi }\end{array}$ & $\begin{array}{c}\text { Ladin Name } \\
\text { Radix Bupleuri } \\
\text { Radix Paeoniae Alba } \\
\text { Radix Angelicae Sinensis } \\
\text { Fructus Aurantii } \\
\text { Radix Paeoniae Rubra } \\
\text { Radix Rehmanniae } \\
\text { Rhizoma Chuanxiong } \\
\text { Herba Leonuri } \\
\text { Cortex Moutan }\end{array}$ & \\
\hline
\end{tabular}

Table 2: Main complaints and incidences.

\begin{tabular}{|c|c|}
\hline Main Complaints & Cases \\
\hline Amenorrhea (continuous missing of menstruation for more than 3 months) & 11 \\
\hline Irregular or regularly prolonged menstruation with intermittent missing of period & 45 \\
\hline No menarche & 1 \\
\hline Infertility & 36 \\
\hline Miscarriages & 8 \\
\hline Depression, Anxiety, Emotional swings, PMT & 21 \\
\hline Obesity & 10 \\
\hline Acne & 15 \\
\hline Hirsutism (excess body and facial hair), & 7 \\
\hline Virilism (secondary male sexual characteristics) & 2 \\
\hline
\end{tabular}




\section{Results}

After receiving combined acupuncture and relevant herbal treatments, or just acupuncture or herbal treatment (according to the patient's preference), the effect rate was determined using the following criterion.

\section{Criterion:}

a) 4.4.1 Cured: no cases are recognized in this column as a longer period of time is needed to follow and analyses cases to make sure they do not recur.

b) Excellent effect:

1) Successful pregnancy;
2) Regular menstruation for at least 3 consecutive months;

3) All relevant symptoms have disappeared.

c) Effective:

1) Regular menstruation, although with some prolonged menstrual cycles (more than 30 days);

2) All relevant symptoms have decreased;

3) The treatment is terminated before the goals (conception/pregnancy, or weight loss) have been achieved, although some relevant symptoms have decrease

4) No effect: the treatment is terminated before the goals are achieved due to financial or other reasons (Table 3).

Table 3: Treating methods and results of 58 cases.

\begin{tabular}{|c|c|c|c|c|c|c|c|c|}
\hline \multirow{2}{*}{ Diagnosis } & \multirow{2}{*}{ Acupuncture } & \multicolumn{3}{|c|}{ Herbal medicine (formulas) } & \multicolumn{3}{|c|}{ Results (\%) } & $\begin{array}{c}\text { Effective rate } \\
\text { (87) }\end{array}$ \\
\cline { 2 - 8 } & & Pills & $\begin{array}{c}\text { Prescribed herb- } \\
\text { al powders }\end{array}$ & $\begin{array}{c}\text { Prescribed } \\
\text { dry herbs* }\end{array}$ & $\begin{array}{c}\text { Excellent } \\
\text { effect }\end{array}$ & Effect & Terminated \\
\hline $\begin{array}{c}\text { Polycystic } \\
\text { Ovary 46 }\end{array}$ & $42(91 \%)$ & $\begin{array}{c}33 \\
(71 \%)\end{array}$ & $\begin{array}{c}5 \\
(11 \%)\end{array}$ & $13(28 \%)$ & $\begin{array}{c}28 \\
(61 \%)\end{array}$ & $\begin{array}{c}12 \\
(26 \%)\end{array}$ & 6 \\
\hline \\
PCOS 12
\end{tabular}

*Some cases are mixed prescribed dry herbs and herbal pills. For instance, prescribed dry herbs are given for the first few weeks/months, upon the return of menstruation herbal pills are given.

\section{Typical Cases}

Case 1: Polycystic ovaries: H.W., 32, medical researcher, had been trying to get pregnant for 3 years without success. Her menstrual cycle was 30 to 50 days; with occasional amenorrhea. An ultrasound scan showed polycystic ovaries although her hormone tests were normal. The patient agreed to regular acupuncture treatment combined with herbal pills for 3 months, with a treating principle of strengthening spleen and replenishing kidneys, warming uterus and expelling the internal cold. She had a full-term pregnancy and gave birth to a health boy.

Case 2: Polycystic ovaries and pregnancy with indications for a miscarriage: M.M-B. 29, IT technician, had been trying to get pregnant for 3 years without success. Her menstrual cycles were prolonged, with occasional amenorrhea. She also complained of fatigue, insomnia, anxiety and constipation. Her gynecologist suspected polycystic ovaries, but blood results did not support the diagnosis. She agreed to regular acupuncture treatment combined with herbal pills, with a treating principle of strengthening spleen and kidneys, warming uterus and dissolving the blood stasis in it. She gradually began to have 27-28 day menstrual cycles, and conceived in the 5 th month of the treatment. Due to a kidney and spleen deficiency, she continued to have irregular vaginal bleeding and low abdominal spasms during her pregnancy. She continued with regular acupuncture and herbal medicine treatment in order to protect the fetus until full term. She gave birth to a healthy boy.

Case 3: Polycystic ovary syndrome with hyperthyroidism: R.H., 23. Clerk, felt very much under stress due to hyperthyroidism after graduating from university and starting work. After regular CWM treatment combined with TCM treatment, her hyperthyroidism was clinically controlled, her TSH returned to normal and all symptoms of hyperthyroidism disappeared. A year later though, she started missing her periods which led to amenorrhea and an increase in weight. She then consulted her GP who diagnosed her with early-stage polycystic ovary syndrome, based on elevated levels of LH and enlarged ovaries. She was given a course of contraceptive pills. Having previously benefited from TCM treatment, when she was suffering from hyperthyroidism, she was willing to give TCM treatment priority over CWM. She decided not to take contraceptive pills and started acupuncture and herbal pill treatment. When she started TCM treatment, she had not had her period for 6 months. After two months of treatment, she had still not got her period, so we switched her to dry herbal prescription. Two months later she started her period. She went on to receive treatment for another 2 months by which time she had had 3 consecutive menstrual cycles. Her blood tests and ultrasound scan came back with normal results. 
Case 4: Polycystic ovary syndrome and husband with low sperm vitality: A.C., 32, nutritionist, had been trying to get pregnant for 3 years without success. Since starting her period at 14 , she had always had menstrual cycles of $32-56$ days. She started taking contraceptive pills when she was 18 and stopped 3 years earlier. Since then she had irregular periods again, and often amenorrhea. The gynecologist diagnosed her with PCOS and prescribed Clomifene for 10 months which made her periods come regularly, but her menstrual cycles were still 5-6 weeks with heavier bleeding, but no pain. Her husband, 27, was found to have a low sperm count with low vitality. The couple was referred to me by their gynecological consultant. Regular acupuncture and herbal pills treatment with a treating principle of replenishing kidney and warming uterus, removing dampness and dissolving blood stasis were given to the wife; herbal pills for promoting the liver Qi, replenishing kidneys and strengthening essence were given to the husband. A month later, the wife had her period after a 28 day cycle and successfully conceived the following month. The couple were very satisfied; the gynecological consultant sent a letter of recognition stating that TCM was very quick in achieving results.

Case 5: Polycystic ovary syndrome and husband with low sperm vitality: M.B., 29, Pakistani-British housewife, suffered from infertility for the past 8 years. She had her first period when she was 16 and since then had never had a proper period. In general, she had her period every 2-3 months, with periods of amenorrhea which lasted from half a year to a couple of years. Obesity and excess of facial and body hair appeared. She had never taken contraceptive pills as she had been trying to get pregnant ever since she got married. Her doctor diagnosed her with polycystic ovary syndrome and told her that IVF treatment was not an option. Her husband was also found to have a low sperm count with low vitality. Their infertility was a source of depression for the couple and so they turned to TCM for help. The wife was given regular acupuncture treatment combined with herbal concentrated powder, guided by the treating principle of strengthening spleen and kidney, and dissolving blood stasis from the uterus. The husband was treated with herbal pills guided by the treating principle of promoting the liver Qi, replenishing kidneys and strengthening essences. After 5 months of treatment, they were accepted for IVF treatment by reproductive medical doctors. I stopped treating her with herbal medicines but kept on giving her regular acupuncture treatment while she was on IVF treatment. After a course of assisted ovulation medication, her eggs were collected and one of them successfully fertilized with her husband's sperm. She gave birth to a healthy boy delivered at full term. The couple were extremely pleased.

\section{Discussion}

Polycystic ovaries have become increasingly widespread in women today. It may be the result of improved and more prevalent diagnostic techniques used in CWM meaning simply that more women are being checked and diagnosed. This tendency is reflected in my study as well: a high percentage (79\%) of cases with PCO and a low percentage $(21 \%)$ of cases with PCOS. Polycystic ovary syndrome is an organic disease with a complex hormonal disorder that gives rise to symptoms such as obesity, acne, hirsutism (excess body and facial hair) and virilism (secondary male sexual characteristics) all of which, apart from infertility cause discomfort and suffering. My experience of PCO and PCOS has led me to the following conclusions:

1) Polycystic ovaries is a milder form of PCOS, existing before PCOS has been diagnosed, meaning it can be classed as a preclinical condition. The practitioner of TCM should pay attention to female patients who complain of irregular or regularly delayed menstrual cycles, as well as amenorrhea. If we recognize cases with PCO, we can offer them specialized treatment, meaning that patients will possibly be cured of PCO, and not go on to develop PCOS. See case 1-3

2) TCM should take the key treating principle of strengthening Qi and warming Yang, removing fluid and dissolving blood stasis in cases of PCO and PCOS. The goal is to regulate the menstrual cycle, that is shorten it to a perfect 28 day one. The effect rate for cases with PCO (87\%) is higher than PCOS (60\%) according to my research.

3) In general, women who have only PCO may not necessarily be infertile. It is possible that a partner's low sperm count is at the root of the couple's inability to get pregnant. It is, therefore, very important to check the partner's sperm when a woman has been diagnosed with PCO. See case 4.

4) Although doctors do not offer IVF treatment for women with PCO or PCOS, TCM treatment can help some patients regulate their hormone levels and state of ovaries to a degree where they meet the IVF criterion. They can then be treated in a safe and effective manner. See case 5.

5) If a woman conceives before she has sufficiently progressed in her treatment, she may be susceptible miscarriage. TCM treatment can help protect the fetus for promoting her to successful end of pregnancy. See case 2.

\section{Conclusion}

It is important to distinguish between polycystic ovaries and polycystic ovary syndrome. TCM is a valuable treatment which should be performed as early as possible in order to give maximum benefit to women with PCO or PCOS.

\section{Acknowledgement}

I will appreciate Ms. Ivana Brasic BA as an English teacher and the mother of a patient with PCOS who offers a help for reviewing and modifying English in this article. 


\section{References}

1. Adam Balen the Polycystic Ovary Syndrome, Organon Laboratories, Cambridge Science Park (2005), Cambridge, UK.

2. Judith Collier (2007) Oxford Handbook of Clinical Specialties---the seventh edition, Oxford University Press, Oxford, UK.

3. Adam Balen (2005) Ovulation Induction, Organon Laboratories, Cambridge Science Park, Cambridge, UK.
4. Guicheng Xia (2000) Infertility Treatment and Menstrual Regulation, People Health Publishing House, Beijing, China.

5. Dan Jiang, Treating Female Infertility by Strengthening the Natural Menstrual Cycle with Acupuncture and Chinese Herbal Medicine, the European Journal of Oriental Medicine 7(6): 28-35.

6. Isabel Stabile (1996) Clinical Obstetrics and Gynecology Springer, New York, USA.

\section{Your next submission with Juniper Publishers will reach you the below assets}

- Quality Editorial service

- Swift Peer Review

- Reprints availability

- E-prints Service

- Manuscript Podcast for convenient understanding

- Global attainment for your research

- Manuscript accessibility in different formats

( Pdf, E-pub, Full Tsext, Audio)

- Unceasing customer service

Track the below URL for one-step submission

https://juniperpublishers.com/online-submission.php 